\title{
Comparison of Underlay and Overlay Spectrum Sharing Strategies in MISO Cognitive Channels
}

\author{
Ricardo Blasco-Serrano ${ }^{\dagger}$, Jing Lv*, Ragnar Thobaben ${ }^{\dagger}$, Eduard Jorswieck*, Adrian Kliks ${ }^{\ddagger}$, and Mikael Skoglund ${ }^{\dagger}$ \\ ${ }^{\dagger}$ School of Electrical Engineering and ACCESS Linnaeus Centre, KTH Royal Institute of Technology, Stockholm, Sweden \\ E-mail: \{ricardo.blasco, ragnar.thobaben, mikael.skoglund\}@ee.kth.se \\ ${ }^{*}$ Communications Theory, Communications Laboratory, Dresden University of Technology, Dresden, Germany \\ E-mail: $\{$ jing.lv, eduard.jorswieck\}@tu-dresden.de \\ ${ }^{\ddagger}$ Chair of Wireless Communications, Poznan University of Technology, Poznan, Poland \\ E-mail: akliks@et.put.poznan.pl
}

\begin{abstract}
We consider an extension of the cognitive radio channel model in which the secondary transmitter has to obtain ("learn") the primary message in a first phase rather than having non-causal knowledge of it. We propose an achievable rate region that combines elements of decode-and-forward relaying with coding for the pure cognitive radio channel model. Moreover, we find the choice of parameters that maximize the secondary rate under a primary rate constraint. Finally, we compare numerically the performance of our system to that of an underlay scheme that combines beamforming, rate splitting, and successive decoding. We observe that although the overlay design provides higher rates, the losses due to the first phase are quite severe. In fact, for the considered scenarios, cleverly designed underlay schemes can provide comparable performance.
\end{abstract}

\section{INTRODUCTION}

Cognitive radio (CR) and opportunistic spectrum sharing are promising concepts for boosting the efficiency of radio spectrum utilization. In the last decade, research on CR has therefore focused on three main spectrum sharing paradigms: underlay, overlay, and interweave [1]. Each of them requires a different level of cognition about the surrounding environment and a different level of sophistication which leads to different challenges.

In this paper we focus on underlay and overlay CR systems and specialize to the scenario where a primary transmitter/receiver pair is willing to share its spectrum with a secondary transmitter/receiver pair, given that the primary rate requirements are satisfied. The most common information theoretical model for underlay CR is the interference channel (IC). The (two-user) IC describes the situation where two independent transmitterreceiver pairs communicate interfering each other. In addition to classical coding strategies for the IC (see e.g. [2], [3]) several authors have studied this scenario with CR constraints (see e.g. [4]-[6]). In particular, multiple-antenna transmit strategies are of special interest for underlay scenarios where interference to the primary receiver must not exceed a given level. Assuming that sufficient channel state information is available at the secondary transmitter, beamforming techniques can be used to control the interference to the primary receiver while maximizing the secondary rate (see e.g. [4], [5]). For the special case of a

Part of this work has been performed in the framework of Network of Excellence ACROPOLIS, which is partly funded by the European Union under its FP7 ICT Objective 1.1 - The Network of the Future. multiple-input/single-output (MISO) secondary system coexisting with a single-input/single-output (SISO) primary system, we investigated in [7] gains in secondary rate that can be obtained by spatial shaping. We showed furthermore that additional gains can be obtained in this setting if rate splitting is used at the secondary transmitter and successive decoding (including the interfering primary message) is performed at the secondary receiver.

In contrast, the cognitive radio channel (also known as IC with degraded message sets) has been used to model overlay CR scenarios. This model differs from the classical IC in that the secondary transmitter has non-causal knowledge of the primary message. Fundamental transmission strategies have been proposed for example in [8]-[10] for the single-antenna case and shown to achieve the capacity for the secondary user for important special cases. In the low interference regime it has been shown in [8], [9] that the optimal strategy at the secondary transmitter consists of combining selfless relaying of the primary message and interference pre-cancellation using dirty paper coding [11] to transmit its own message. In contrast, the capacity of part of the strong interference regime was obtained in [10] using superposition coding and interference decoding. In our recent work [12], we adopted the strategy from [8], [9] to the case where a MISO secondary system coexists in the spectrum of a SISO primary system and discussed joint optimization of beamforming and power allocation to maximize the secondary rate while satisfying the primary rate constraint.

A comparison of fundamental strategies for underlay and overlay CR transmission suggests that non-causal knowledge of the primary message at the secondary transmitter leads to a significant increase in secondary rate. However, a critical point of the overlay CR concept is how knowledge of the primary message can be obtained. In [13], [14], it is suggested that a $\mathrm{CR}$ can learn the primary message by overhearing primary ARQ transmissions and utilize knowledge of the message to perform spectrum overlay during retransmissions of the primary system. As an alternative [15] and [16] consider explicit cooperation of the primary and secondary systems. In any case, learning the primary message requires the secondary transmitter to be passive for a certain duration in time, and it leads therefore inevitably to a rate loss for the secondary system which may lower the attractiveness of overlay strategies. 
Unfortunately, fundamental studies of overlay transmissions do not account for this rate loss, and the best known achievable rates may therefore be too optimistic. Motivated by this observation, we consider in this paper an extension of the $\mathrm{CR}$ channel model that takes into account the initial transmission phase for exchanging the primary message as well. For this extended CR channel model, we propose an achievable transmission scheme that combines decode-and-forward (DF) relaying [17], [18] with the transmission strategy for the CR channel proposed in [8], [9]. Achievable rates derived for the proposed scheme are then used to compare the performance of underlay and overlay strategies using a simple geometrical model for the relative positions of the nodes. As in [12], we consider the coexistence of a SISO primary system and a MISO secondary system and optimize beamforming and power allocation to maximize the secondary rate under a primary rate constraint. As [15], [16], we consider in this paper explicit cooperation between the primary and secondary transmitters. However, in contrast to [15], [16] which use repetition coding at the relays, our relaying strategy is based on binning. The design goal in [16] is furthermore, to help the primary transmitter to transmit as fast as possible in order to maximize the number of unoccupied channel uses which can then be used by the secondary system.

The remainder of this paper is organized as follows: In Section II, we introduce the system model along with the notation. In Section III we formulate the proposed transmission strategy and the problem statement. In Section IV we find the optimal choice of system parameters for our overlay transmission strategy in extended cognitive radio channels. In Section V we evaluate numerically the performance of the strategy based on a simple geometrical model for the relative positions of the nodes. Moreover, we compare it against an underlay strategy that controls the interference at the primary receiver. Finally, in Section VI we conclude our work.

\section{Preliminaries}

\section{A. Notation}

Column vectors and matrices are represented in lower case and upper case boldface letters, respectively. $|\cdot|$ is the absolute value of a scalar, $\|\cdot\|$ is the Frobenius norm of a vector or matrix, and $(\cdot)^{H}$ stands for Hermitian transpose. $\Pi_{\boldsymbol{X}} \triangleq \boldsymbol{X}\left(\boldsymbol{X}^{H} \boldsymbol{X}\right)^{-1} \boldsymbol{X}^{H}$ denotes the orthogonal projection onto the column space of $\boldsymbol{X}$, and $\Pi_{\boldsymbol{X}}^{\perp} \triangleq \boldsymbol{I}-\Pi_{\boldsymbol{X}}$, where $\boldsymbol{I}$ is the identity matrix, denotes the orthogonal projection onto the orthogonal complement of the column space of $\boldsymbol{X}$. In this paper all logarithms are taken to the base of 2 and all rates are expressed in bits.

\section{B. System Model}

As in [7], [12], we consider a SISO primary system which is willing to share its channel with a MISO secondary system with $N$ antennas. We consider transmission in two phases where the first phase is used to inform the secondary system about the primary message. The second phase corresponds to the set-up which is known as the cognitive radio channel. We assume that the secondary transmitter cannot transmit and receive at the same time (i.e., we consider a half duplex constraint) and it uses its $N$ antennas for both transmission and reception. The $t^{t h}$ received sample (see Fig. 1) from the matched-filtered complex baseband model in the first phase is

$$
\begin{aligned}
y_{1}^{(1)}(t) & =h_{11} x_{1}^{(1)}(t)+n_{1}(t) \\
\boldsymbol{y}_{s t}(t) & =\boldsymbol{h}_{t t} x_{1}^{(1)}(t)+\boldsymbol{n}_{s t}(t)
\end{aligned}
$$

where $x_{1}^{(1)}(t)$ is the signal transmitted by the primary transmitter and $y_{1}^{(1)}(t)$ and $\boldsymbol{y}_{s t}(t)$ are the channel-attenuated noise-corrupted observations at the primary receiver and secondary transmitter, respectively. Similarly for the second phase

$$
\begin{aligned}
& y_{1}^{(2)}(t)=h_{11} x_{1}^{(2)}(t)+\boldsymbol{h}_{21}^{H} \boldsymbol{x}_{2}^{(2)}(t)+n_{1}(t) \\
& y_{2}^{(2)}(t)=h_{12} x_{1}^{(2)}(t)+\boldsymbol{h}_{22}^{H} \boldsymbol{x}_{2}^{(2)}(t)+n_{2}(t)
\end{aligned}
$$

where $x_{1}^{(2)}(t)$ and $\boldsymbol{x}_{2}^{(2)}(t)$ are the signals transmitted by the primary and secondary transmitters and $y_{1}^{(2)}(t)$ and $y_{2}^{(2)}(t)$ are the observations at the respective receivers. This model is illustrated in Figure 1.

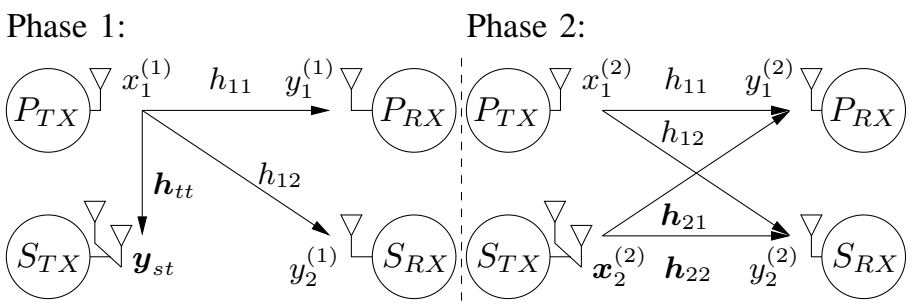

Fig. 1. Illustration of the two-phase transmission scheme.

The noise processes on all channels are modelled by independent complex additive white Gaussian noise (AWGN) processes with unit noise variance: $n_{1}, n_{2} \sim \mathcal{C N}(0,1), \boldsymbol{n}_{s t} \sim \mathcal{C N}(0, \boldsymbol{I})$. We denote by $h_{i j}$ the channel gain (vector of channel gains for multiple antennas) from transmitter $i \in\{1,2\}$ to receiver $j \in\{1,2\}$, which we assume to remain constant during the two phases. The channel gain vector in the first phase between transmitters is denoted as $\boldsymbol{h}_{t t}$. We model the channel gains as $h_{11} \sim \mathcal{C N}\left(0, \sigma_{11}^{2}\right), h_{12} \sim \mathcal{C N}\left(0, \sigma_{12}^{2}\right), h_{t t} \sim \mathcal{C N}\left(0, \sigma_{t t}^{2} \boldsymbol{I}\right)$, $\boldsymbol{h}_{21} \sim \mathcal{C N}\left(0, \sigma_{21}^{2} \boldsymbol{I}\right)$, and $\boldsymbol{h}_{22} \sim \mathcal{C N}\left(0, \sigma_{22}^{2} \boldsymbol{I}\right)$. We assume that all nodes have perfect channel knowledge on all links.

The entire transmission is carried out over $n$ channel uses, $k$ channel uses are consumed during the first transmission phase, and $(n-k)$ channel uses are used during the second phase. The fraction of channel uses in the first and the second phases are accordingly given by $\alpha=k / n$ and $1-\alpha$, respectively.

\section{Transmission Strategies and Problem FORMULATION}

For each of the CR paradigms we will consider a different transmission strategy. In the case of underlay CR we will use the strategy from [7] that combines power allocation, beamforming, and rate splitting (whenever possible) at the secondary transmitter and successive decoding at the secondary receiver. We introduce our strategy for overlay CR in the following section. 


\section{A. Overlay Transmission Strategy}

Our strategy relies on DF principles [17] to adapt the overlay CR strategy from [12] to the extended cognitive radio channel model where the secondary transmitter has to acquire the primary message.

We assume that the primary user has a target rate $R_{1}^{\star}$. During the first phase, which has relative duration equal to $\alpha$, the primary transmitter uses a code $\mathcal{C}_{0}$ with rate $R_{0}=\frac{R_{1}^{\star}}{\alpha}$ to broadcast its message to both the primary receiver and the secondary transmitter using power $P_{1}^{(1)}$. We define the rates

$$
\begin{aligned}
R_{1}^{(1)} & =\alpha \log \left(1+\left|h_{11}\right|^{2} P_{1}^{(1)}\right), \\
R_{t} & =\alpha \log \left(1+\left\|\boldsymbol{h}_{t t}\right\|^{2} P_{1}^{(1)}\right) .
\end{aligned}
$$

Clearly only those rates $R_{1}^{\star}$ satisfying

$$
R_{t} \geq R_{1}^{\star}
$$

can be used to ensure decodability at the secondary transmitter at the end of the first phase. Assuming that

$$
\left\|\boldsymbol{h}_{t t}\right\|>\left|h_{11}\right|,
$$

after the first phase the primary receiver only has partial knowledge (i.e. its observation) about the message sent by the primary transmitter. In the second phase the primary and secondary transmitters cooperate to resolve this ambiguity at the primary receiver. To do so the code $\mathcal{C}_{0}$ is subdivided into disjoint (lowerrate) sub-codes $\mathcal{C}_{i}$ (bins), which are designed to be good channel codes. That is, each codeword of $\mathcal{C}_{0}$ belongs to a sub-code $\mathcal{C}_{i}$. The index $i$ of the sub-code containing the codeword sent in the first phase is transmitted cooperatively by the primary transmitter (using power $P_{1}^{(2)}$ ) and the secondary transmitter (using a fraction $p_{a}$ of its available power $P_{2}$ ). Knowing this index the primary receiver can decode its message based on its channel observation from the first phase by considering only codewords from the (low-rate) sub-code $\mathcal{C}_{i}$. In addition, the secondary transmitter employs the remaining power $p_{b}$ for its own private communication, using dirty paper coding to precode the message. Relayed and private messages are broadcasted by the secondary transmitter using independent beamformers $\boldsymbol{w}_{1}$ and $\boldsymbol{w}_{2}$, respectively. We define the rates

$$
\begin{aligned}
& R_{1}^{(2)}=(1-\alpha) \log \left(1+\frac{\left|h_{11} \sqrt{P_{1}^{(2)}}+\boldsymbol{h}_{21}^{H} \boldsymbol{w}_{1} \sqrt{\frac{p_{a}}{1-\alpha}}\right|^{2}}{1+\left|\boldsymbol{h}_{21}^{H} \boldsymbol{w}_{2}\right|^{2} \frac{p_{b}}{1-\alpha}}\right), \\
& R_{2}=(1-\alpha) \log \left(1+\left|\boldsymbol{h}_{22}^{H} \boldsymbol{w}_{2}\right|^{2} \frac{p_{b}}{1-\alpha}\right)
\end{aligned}
$$

that upper bound the cooperative transmission rate (i.e. transmission of the bin index) and the secondary rate achievable by our strategy in the second phase. Note that the powers are scaled up to take into account the duration of the second phase. Using DF relaying arguments (see e.g. [17], [18]) it is possible to show that the largest communication rate for the primary users is given by

$$
R_{1}=R_{1}^{(1)}+R_{1}^{(2)} .
$$

The boundary of the rate region achievable with our transmission strategy is $\mathcal{R}=\left(R_{1}, R_{2}\right)$ for any given (valid) choice of beamformers, phase splitting, and power allocation that ensures decodability of the primary message at the secondary transmitter after the first phase. Clearly we are interested in the choice of these parameters that gives the largest achievable rate region. We study this in the following section.

Remark: It is possible to increase the primary rate by performing rate splitting and superposition coding at the primary transmitter (see e.g. [18]). For simplicity we do not consider this here.

Remark: We recover the half-duplex DF relaying rates by setting $R_{2}=0$ in our achievable rate region (Eq. (14) in [18] with $\beta=1$ ).

\section{B. Problem Formulation}

We are interested in finding the choice of beamformers, phase splitting, and power allocation that maximizes the secondary rate $R_{2}$ while ensuring a target rate $R_{1}^{\star}$ for the primary user pair under average power constraints $P_{1}$ and $P_{2}$ at the primary and secondary transmitters, respectively. This is formulated mathematically as

$$
\begin{aligned}
& \max _{\substack{\alpha \\
\boldsymbol{w}_{1}, p_{a} \\
\boldsymbol{w}_{2}, p_{b} \\
P_{1}^{(1)}, P_{1}^{(2)}}}(1-\alpha) \log \left(1+\left|\boldsymbol{h}_{22}^{H} \boldsymbol{w}_{2}\right|^{2} \frac{p_{b}}{1-\alpha}\right) \\
& \text { subject to: } \\
& \quad R_{t} \geq R_{1}^{\star}, \\
& R_{1} \geq R_{1}^{\star}, \\
& \quad\left\|\boldsymbol{w}_{1}\right\|=\left\|\boldsymbol{w}_{2}\right\|=1, \\
& \quad \alpha P_{1}^{(1)}+(1-\alpha) P_{1}^{(2)} \leq P_{1}, P_{1}^{(1)} \geq 0, P_{1}^{(2)} \geq 0, \\
& p_{a}+p_{b} \leq P_{2}, p_{a} \geq 0, p_{b} \geq 0, \\
& 0<\alpha<1 .
\end{aligned}
$$

Remark: Constraint (3b) enforces decodability of the primary message at the secondary transmitter. This might be too restrictive in some cases, e.g. if $\left|h_{11}\right|>|| \boldsymbol{h}_{t t}||$. This is a wellknown side effect of DF strategies and may motivate other approaches (e.g. based on compress-and-forward) [17], [18]. In the following we disregard this issue and focus on the problem as described in (3). In the simulations we will allow the secondary user to transmit even if (3b) is not satisfied as long as $R_{1}^{(1)} \geq R_{1}^{\star}$.

\section{System OptimizATION}

In this Section we reduce the system optimization problem defined in (3) to a search over two parameters. We shall make use of the following Lemma in our arguments

Lemma 1. The function

$$
\beta \log \left(1+\frac{P}{\beta}\right)
$$

defined for $\beta \in(0,1]$ and $P>0$ is strictly increasing in $\beta$.

This Lemma expresses the well-known fact that, due to the logarithmic nature of Gaussian-like rate expressions, for a 
fixed average power the best option is to use all the available bandwidth at low power rather than concentrating a high power signal on a smaller band.

Proposition 1. The solution to the optimization problem makes use of all the available power at the primary and secondary transmitters. That is:

1) $p_{a}+p_{b}=P_{2}$.

2) $\alpha P_{1}^{(1)}+(1-\alpha) P_{1}^{(2)}=P_{1}$,

Proof: First we prove Statement 1: Let the set of parameters $\left(\alpha, P_{1}^{(1)}, P_{1}^{(2)}, p_{a}, p_{b}, \boldsymbol{w}_{1}, \boldsymbol{w}_{2}\right)$ attain the optimum and be such that

$$
p_{a}+p_{b}<P_{2} .
$$

The relationship between $p_{a}$ and $p_{b}$ to keep $R_{1}^{(2)}$ constant can be expressed as a continuous function of $p_{a}$. Therefore we can find a power $\tilde{p}_{b}>p_{b}$ with sufficiently small $\tilde{p}_{b}-p_{b}>0$ such that the required increase in power $\tilde{p}_{a} \geq p_{a}$ to keep $R_{1}^{(2)}$ unchanged (and hence satisfy (3c)) does not violate constraint (3f). However, using a larger power $\tilde{p}_{b}$ increases the rate $R_{2}$ which contradicts our assumption that the set of parameters solved the optimization problem.

We now prove Statement 2. Again let the set of parameters $\left(\alpha, P_{1}^{(1)}, P_{1}^{(2)}, p_{a}, p_{b}, \boldsymbol{w}_{1}, \boldsymbol{w}_{2}\right)$ attain the optimum and be such that

$$
\alpha P_{1}^{(1)}+(1-\alpha) P_{1}^{(2)}<P_{1} .
$$

Then we can find a larger power for the first phase $\tilde{P}_{1}^{(1)}>P_{1}^{(1)}$ such that (4) and constraints (3b) and (3c) are satisfied with strict inequality. Moreover note that (4), $R_{1}$, and $R_{t}$ are continuous in $\alpha$. Therefore we can shorten the duration of the first phase to $\tilde{\alpha}$ with $\alpha-\tilde{\alpha}>0$ sufficiently small while ensuring that constraints (3b), (3c), and (3e) are still satisfied. However, from Lemma 1 we know that the rate $R_{2}$ is increased by having a longer second phase. Hence we have again contradicted our initial statement.

Proposition 2. Considering the problem in Section III-B, for certain $\alpha \in(0,1)$ and $P_{1}^{(1)} \in\left(0, \frac{P_{1}}{\alpha}\right]$,

1) if $R_{1}^{(1)} \geq R_{1}^{\star}$,

$$
\begin{gathered}
\boldsymbol{w}_{2}=\frac{\boldsymbol{h}_{22}}{\left\|\boldsymbol{h}_{22}\right\|}, \\
p_{b}=P_{2} .
\end{gathered}
$$

2) if $R_{1}^{(1)}<R_{1}^{\star}$, (3) is solved by

$$
\begin{gathered}
\boldsymbol{w}_{1}=\frac{h_{11}}{\left|h_{11}\right|} \frac{\boldsymbol{h}_{21}}{\left\|\boldsymbol{h}_{21}\right\|}, \\
\boldsymbol{w}_{2}=\sqrt{\lambda_{2}} \frac{\Pi_{\boldsymbol{h}_{21}} \boldsymbol{h}_{22}}{\left\|\Pi_{\boldsymbol{h}_{21}} \boldsymbol{h}_{22}\right\|}+\sqrt{1-\lambda_{2}} \frac{\Pi_{\boldsymbol{h}_{21}}^{\perp} \boldsymbol{h}_{22}}{\left\|\Pi_{\boldsymbol{h}_{21}}^{\perp} \boldsymbol{h}_{22}\right\|}, \\
p_{b}=P_{2}-p_{a},
\end{gathered}
$$

where

$$
\lambda_{2}= \begin{cases}\lambda_{M R T} & \text { if } \lambda_{M R T} \leq \frac{z(1-\alpha)}{\left\|\boldsymbol{h}_{21}\right\|^{2}\left(P_{2}-p_{a}\right)} \\ \frac{z(1-\alpha)}{\left\|\boldsymbol{h}_{21}\right\|^{2}\left(P_{2}-p_{a}\right)} & \text { otherwise }\end{cases}
$$

$$
\begin{gathered}
\lambda_{M R T}=\frac{\left\|\Pi_{\boldsymbol{h}_{21}} \boldsymbol{h}_{22}\right\|^{2}}{\left\|\boldsymbol{h}_{22}\right\|^{2}}, \\
z=\frac{\left(\left|h_{11}\right| \sqrt{P_{1}^{(2)}}+\| \boldsymbol{h}_{21}|| \sqrt{\frac{p_{a}}{1-\alpha}}\right)^{2}}{2^{\frac{R_{1}^{\star}-R_{1}^{(1)}}{1-\alpha}}-1}-1 \geq 0, \\
P_{1}^{(2)}=\frac{P_{1}-\alpha P_{1}^{(1)}}{1-\alpha},
\end{gathered}
$$

for certain $p_{a} \in\left[0, P_{2}\right]$.

The optimal objective value in (3a) can be obtained by searching over $\alpha, P_{1}^{(1)}$, and $p_{a}$, while satisfying (3b) and (9).

Proof: Case 1 is straightforward. For Case $2 \boldsymbol{w}_{1}$ is chosen as in (5) to maximize the desired signal power at the primary receiver in the second phase, or equivalently $R_{1}^{(2)}$. Given $\alpha$ and $P_{1}^{(1)}$, (3) is equivalent to

$$
\begin{aligned}
& \max _{\boldsymbol{w}_{2}, p_{a}} \min \left(\left(P_{2}-p_{a}\right)\left|\boldsymbol{h}_{22}^{H} \boldsymbol{w}_{2}\right|^{2}, \frac{z(1-\alpha)\left|\boldsymbol{h}_{22}^{H} \boldsymbol{w}_{2}\right|^{2}}{\left|\boldsymbol{h}_{21}^{H} \boldsymbol{w}_{2}\right|^{2}}\right) \\
& \text { s.t. }\left\|\boldsymbol{w}_{2}\right\|=1,0 \leq p_{a} \leq P_{2} .
\end{aligned}
$$

Similar to the proof of Proposition 1 in [7], given $p_{a}$ the optimal $\boldsymbol{w}_{2}$ can be parametrized as in (6).

The following proposition allows us to reduce the search space to only two parameters.

Proposition 3. The set of parameters that attains the maximum $\left(\alpha, P_{1}^{(1)}, P_{1}^{(2)}, p_{a}, p_{b}, \boldsymbol{w}_{1}, \boldsymbol{w}_{2}\right)$ in (3) satisfies constraint (3c) with equality if $\| \boldsymbol{h}_{t t}||>\left|h_{11}\right|$.

Proof: We divide the proof in different cases and show each of them by contradiction.

a) $p_{a}>0$ : Assume that the set of parameters that maximize (3a) yield

$$
R_{1}^{(1)}+R_{1}^{(2)}>R_{1}^{\star}
$$

Then there exists $\tilde{p}_{a}<p_{a}$ that yields a rate in the second phase $\tilde{R}_{1}^{(2)}<R_{1}^{(2)}$ such that

$$
R_{1}^{(1)}+\tilde{R}_{1}^{(2)}>R_{1}^{\star}
$$

Now let $\tilde{p}_{b}=P_{2}-\tilde{p}_{a}>p_{b}$. Without loss of generality we can write

$$
\sqrt{p_{b}} \boldsymbol{w}_{2}=\sqrt{p_{b}^{\perp}} \boldsymbol{w}_{\perp}+\sqrt{\hat{p}_{b}} \hat{\boldsymbol{w}}
$$

where $p_{b}=p_{b}^{\perp}+\hat{p}_{b}, \boldsymbol{w}_{\perp}$ is a unitary vector in an orthogonal direction to $\boldsymbol{h}_{21}$, and $\hat{\boldsymbol{w}}$ is a unitary vector orthogonal to $\boldsymbol{w}_{\perp}$. We can use the additional power $\tilde{p}_{b}-p_{b}$ in the direction of $\boldsymbol{w}_{\perp}$ to obtain a higher rate $R_{2}$ without increasing the interference at the primary receiver (i.e. without violating (3c)). However, this contradicts our assumption that the set of parameters solved the optimization problem.

The preceding argument fails if $\boldsymbol{h}_{21}$ and $\boldsymbol{h}_{22}$ are collinear. However, in this case we can proceed as in the proof of the first part of Proposition 1. 
b) $p_{a}=0$ and $P_{1}^{(1)}<\frac{P_{1}}{\alpha}$ : Again assume that the set of parameters that maximize (3a) yield

$$
R_{1}(\alpha)=R_{1}^{(1)}+R_{1}^{(2)}>R_{1}^{\star}
$$

where we have made explicit the dependency on $\alpha$.

If (3b) is satisfied with equality then we can find $\tilde{P}_{1}^{(1)}>P_{1}^{(1)}$ such that

$$
\tilde{\alpha}=\frac{R_{1}^{\star}}{\log \left(1+\left|h_{11}\right|^{2} \tilde{P}_{1}^{(1)}\right)}<\alpha
$$

is a valid duration for the first phase. Note that this function defining $\tilde{\alpha}$ is continuous in $\tilde{P}_{1}^{(1)}$. Using Proposition 1 we can write the power used by the primary transmitter in the second phase as

$$
\tilde{P}_{2}^{(2)}=\frac{P_{1}-\tilde{\alpha} \tilde{P}_{1}^{(1)}}{1-\tilde{\alpha}}
$$

so that $R_{1}(\tilde{\alpha})$ is also continuous in $\tilde{\alpha}$. Using continuity arguments we see that we can choose a sufficiently small

$$
\tilde{P}_{1}^{(1)}-P_{1}^{(1)}>0
$$

that yields a sufficiently small $\alpha-\tilde{\alpha}>0$ such that (3c) is still satisfied. However, from Lemma 1 we know that the rate $R_{2}$ is increased by having a longer second phase. Hence we have again contradicted our initial statement.

The case where (3b) is a strict inequality is even simpler because we can directly find $\tilde{\alpha}<\alpha$ such that constraint (3b) is still satisfied and then use the same arguments as above.

c) $p_{a}=0$ and $P_{1}^{(1)}=\frac{P_{1}}{\alpha}$ : Note that in this case $R_{1}^{(2)}=0$ so that for any $R_{1}^{\star}>0$ the primary receiver has to decode its message based on the observation from the first phase alone. If $\left\|\boldsymbol{h}_{t t}\right\|>\left|h_{11}\right|$ then satisfying (3c) ensures that (3b) is also satisfied. From Lemma 1 we know that in this case (with $p_{a}=0$, $p_{b}=P_{2}$, and $P_{1}^{(2)}=0$ ) the largest secondary rate is obtained when the second phase is longest, or equivalently when the first phase is shortest. Clearly, this implies that $R_{1}^{(1)}=R_{1}^{\star}$.

\section{Numerical Evaluation}

\section{A. Geometrical Model}

To present our results we will use the simple geometrical model in Fig. 2 in which the different nodes are placed on a plane. The relative positioning of the nodes is summarized by the distance between each pair of nodes. Using the distance $d_{i, j}$ between two nodes we model the block flat fading channel coefficient between them as

$$
h_{i j}=\frac{1}{\sqrt{d_{i, j}^{p}}} \tilde{h}_{i j}
$$

where $p$ is the path loss exponent and $\tilde{h}_{i j} \sim \mathcal{C N}(0,1)$. In the case of channel vectors each of the coefficients is independently modelled as in (11).

For convenience we normalize all distances with respect to the distance between the primary users (i.e. $d_{11}=1$ ). We will consider the square surface $\{(x, y): x \in[0,1], y \in[0,1]\}$ and vary the position of the secondary nodes (relative to the primary

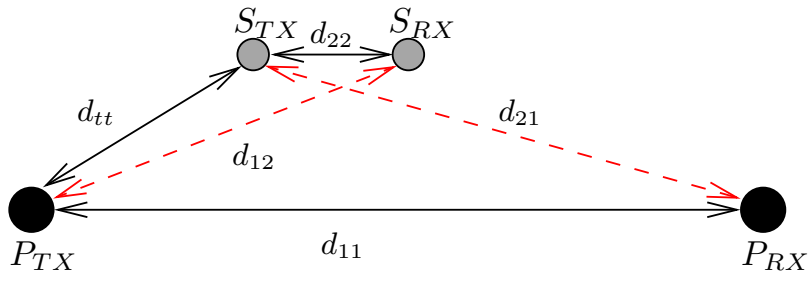

Fig. 2. Geometrical model.

nodes) over a regular square grid of size $11 \times 11$. That is, we will move the secondary transmitter and receiver over this grid, always parallel to the primary user pair (as in Fig. 2), while the primary transmitter and receiver will be fixed at positions $(0,0.5)$ and $(1,0.5)$, respectively. All our results consider $d_{22}=1 / 4$ while the remaining distances $d_{12}, d_{21}$, and $d_{t t}$ vary as described before. This models a secondary middle-range communication in the presence of primary users.

\section{B. Numerical Results}

We now compare the overlay strategy introduced in Section III-A for the extended cognitive radio channel to the underlay strategy from [7], which is based on beamforming and rate splitting at the secondary transmitter and interference decoding at the secondary receiver, whenever possible.

We have chosen $P_{1}=10 \mathrm{~dB}, P_{2}=5 \mathrm{~dB}$ (relative to a unit noise variance, as described in Section II-B), $N=2$ antennas at the secondary transmitter, and path loss exponent $p=3$. The primary system has a target rate $R_{1}^{\star}$ which corresponds to $75 \%$ of its point-to-point Shannon capacity. Each operating point represents the average behaviour over $10^{4}$ random samples.

Figure 3 shows that for most of the square region the overlay system yields more frequently a higher instantaneous rate than the underlay system. The exception to this is the area where the distance between transmitters $d_{t t}$ roughly exceeds the distance between primary users $d_{11}$. The reason is simple: the secondary user cannot transmit until it has decoded the message or the primary users have finished their communication. This waiting time can be quite large for these positions (i.e. $\alpha \approx 1$ ) thus lowering the rate obtained by secondary overlay users. In contrast, an underlay system can transmit from the beginning just taking into account the induced interference.

Rather surprisingly, having the transmitters collocated does not give the overlay system its largest advantage over the underlay system. Note that in this case for low and moderate values of $d_{12}$ the interference at the secondary receiver can be quite large. This does not affect the overlay system because it can pre-cancel the interference using dirty paper coding. In addition, the learning phase is often quite short making this the ideal scenario for the overlay system. However, the underlay approach can increase its rate as well by exploiting the strong interference using rate splitting and successive decoding. We have observed that if the underlay scheme does not implement such functionalities the picture changes dramatically: in that case the overlay system obtains the largest gains compared to the underlay one when the two transmitters are collocated, as one would expect. 


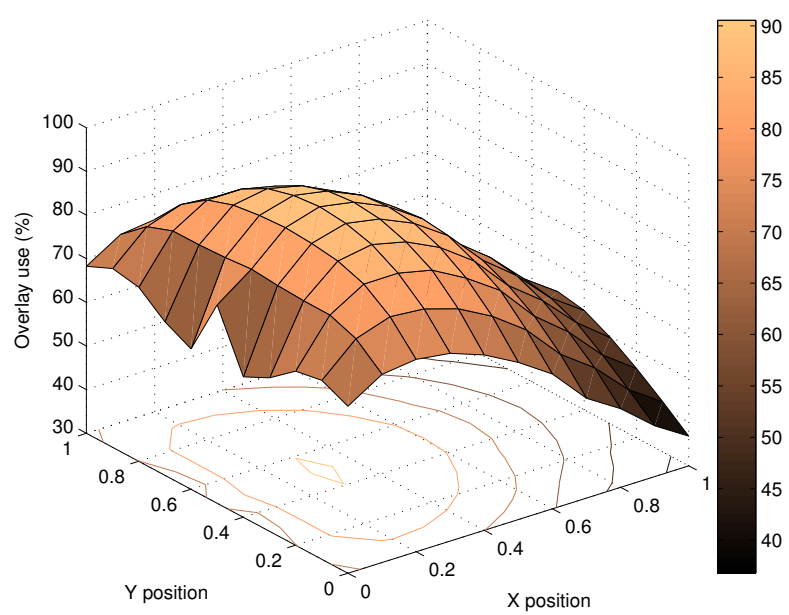

Fig. 3. Instantaneous advantage of the overlay system: percentage of channel realizations that yield a larger instantaneous overlay rate for secondary nodes distributed according to the grid.

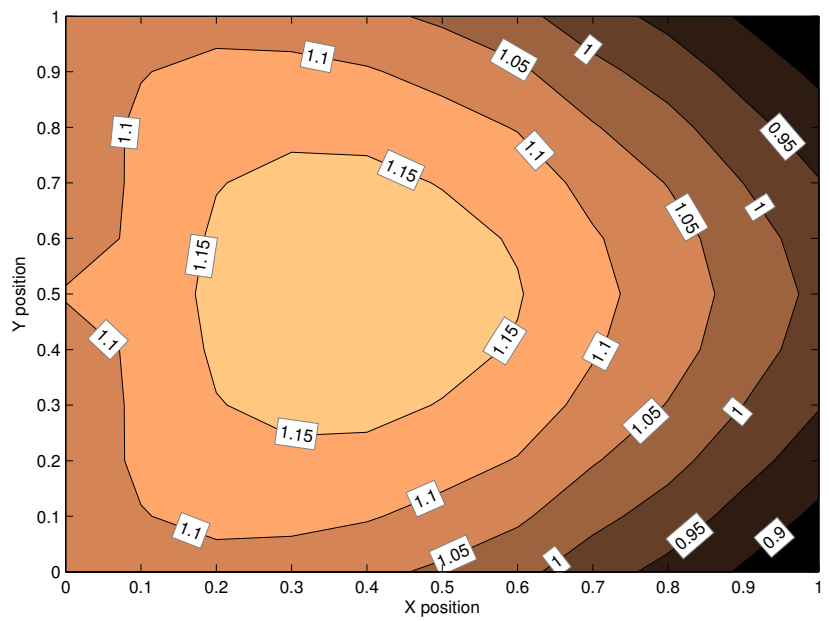

Fig. 4. Average advantage of the overlay system: contour plot for the ratio of average rates $R_{\text {overlay }} / R_{\text {underlay }}$ for secondary nodes distributed according to the grid.

Figure 4 shows the average gain obtained by using the overlay strategy when compared to the underlay, measured as the ratio

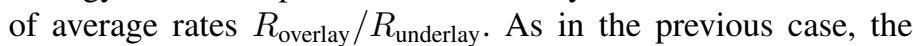
overlay system exhibits an advantage for most of the region (except when the transmitters are farthest apart). However, the gain is not so large, only up to $15 \%$ higher average rate. Apparently, the loss in rate due to the initial (i.e. learning) phase is quite considerable. This brings the performance much closer to that of an underlay system that combats the interference created by the primary by deploying rate splitting and successive decoding as the one in [7]. This is in contrast to the ideal case in which the secondary transmitter has non-causal knowledge of the primary message [12]. In that case gains over $50 \%$ for similar operating conditions were possible.

\section{CONCLUSION}

In this paper we have studied overlay cognitive radio channels that include a learning phase in which the secondary transmitter gains knowledge of the primary message. First, we have designed a transmission strategy for such channels based on decode-andforward principles that combines private communication with selfless relaying of the primary message to mitigate the interference created. Then we have found the choice of parameters that maximizes the secondary rate while ensuring a minimum rate for the primary users.

Finally, we have compared our design to an underlay system whose interaction with the primary users is restricted to controlling the interference it induces on them. We have observed that, although superior in most cases, the overlay approach suffers a significant rate loss due to the learning phase. We have also seen that cleverly designed underlay systems can approach the performance of the overlay scheme by employing interference decoding methods.

\section{REFERENCES}

[1] A. Goldsmith, S.A. Jafar, I. Maric, and S. Srinivasa, "Breaking spectrum gridlock with cognitive radios: An information theoretic perspective," Proc. IEEE, vol. 97, no. 5, pp. 894-914, May 2009.

[2] A. Carleial, "Interference channels," IEEE Transactions on Information Theory, vol. 24, no. 1, pp. 60-70, Jan. 1978.

[3] Han. T.S. and K. Kobayashi, "A new achievable rate region for the interference channel," IEEE Transactions on Information Theory, vol. 27, no. 1, pp. 49-60, Jan. 1981.

[4] R. Zhang and Y.-C. Liang, "Exploiting multi-antennas for opportunistic spectrum sharing in cognitive radio networks," IEEE Journal on Selected Topics in Signal Processing, vol. 2, no. 1, pp. 88-102, Feb. 2008.

[5] R. Zhang, Y.-C. Liang, and S. Cui, "Dynamic resource allocation in cognitive radio networks," IEEE Signal Processing Magazine, vol. 27, no. 3, pp. 102-114, May 2010.

[6] W. Zhang and U. Mitra, "Spectrum shaping: a new perspective on cognitive radio-part I: coexistence with coded legacy transmission," IEEE Transactions on Communications, vol. 58, no. 6, pp. 1857-1867, June 2010.

[7] J. Lv and E. A. Jorswieck, "Spatial shaping in cognitive system with coded legacy transmission," in Proc. of International ITG Workshop on Smart Antennas (WSA), Feb. 2011.

[8] W. Wu, S. Vishwanath, and A. Arapostathis, "Capacity of a class of cognitive radio channels: Interference channels with degraded message sets," IEEE Transactions on Information Theory, vol. 53, no. 11, pp. 43914399, Nov. 2007.

[9] A. Jovicic and P. Viswanath, "Cognitive radio: An information-theoretic perspective," IEEE Transactions on Information Theory, vol. 55, no. 9, pp. 3945-3958, Sept. 2009.

[10] I. Maric, R. D. Yates, and G. Kramer, "Capacity of interference channels with partial transmitter cooperation," IEEE Transactions on Information Theory, vol. 53, no. 10, pp. 3536-3548, Oct. 2007.

[11] M. Costa, "Writing on dirty paper (corresp.)," IEEE Transactions on Information Theory, vol. 29, no. 3, pp. 439-441, May 1983.

[12] J. Lv, R. Blasco-Serrano, E.A. Jorswieck, R. Thobaben, and A. Kliks, "Optimal beamforming in MISO cognitive channels with degraded message sets," in Proc. IEEE Wireless Communications and Networking Conf. (WCNC), 2012.

[13] N. Michelusi, O. Simeone, M. Levorato, P. Popovski, and M. Zorzi, "Optimal cognitive transmission exploiting redundancy in primary ARQ process," in Proc. Workshop on Inf. Theory and App. (ITA), Feb. 2011.

[14] R. A. Tannious and A. Nosratinia, "Cognitive radio protocols based on exploiting hybrid ARQ retransmissions," IEEE Transactions on Wireless Communications, vol. 9, no. 9, pp. 2833-2841, Sept. 2010.

[15] Y. Han, A. Pandharipande, and S. Ting, "Cooperative decode-and-forward relaying for secondary spectrum access," IEEE Transactions on Wireless Communications, vol. 8, no. 10, pp. 4945-4950, Oct. 2009.

[16] Y. Li, P. Wang, and D. Niyato, "Optimal power allocation for secondary users in cognitive relay networks," in Proc. IEEE Wireless Communications and Networking Conf. (WCNC), Mar. 2011, pp. 862-867.

[17] T. Cover and A. El Gamal, "Capacity theorems for the relay channel," IEEE Transactions on Information Theory, vol. 25, no. 5, pp. 572-584, Sept. 1979.

[18] A. Høst-Madsen and J. Zhang, "Capacity bounds and power allocation for wireless relay channels," IEEE Transactions on Information Theory, vol. 51, no. 6, pp. 2020-2040, June 2005. 